\title{
Interim Guidelines for the Evaluation and Testing of Infants with Possible Congenital Zika Virus Infection — United States, 2016
}

\author{
J. Erin Staples, MD, $\mathrm{PhD}^{1}$; Eric J. Dziuban, $\mathrm{MD}^{2}$; Marc Fischer, $\mathrm{MD}^{1}$; Janet D. Cragan, MD ${ }^{3}$; Sonja A. Rasmussen, MD ${ }^{4}$; \\ Michael J. Cannon, $\mathrm{PhD}^{3}$; Meghan T. Frey, MPH${ }^{3}$; Christina M. Renquist, MPH ${ }^{3}$; Robert S. Lanciotti, PhD ${ }^{1}$; Jorge L. Muñoz, PhD ${ }^{1}$; \\ Ann M. Powers, $\mathrm{PhD}^{1}$; Margaret A. Honein, $\mathrm{PhD}^{3}$; Cynthia A. Moore, $\mathrm{MD}, \mathrm{PhD}^{3}$
}

On January 26, 2016, this report was posted as an MMWR Early Release on the MMWR website (http://www.cdc.gov/mmwr). CDC has developed interim guidelines for health care providers in the United States who are caring for infants born to mothers who traveled to or resided in an area with Zika virus transmission during pregnancy. These guidelines include recommendations for the testing and management of these infants. Guidance is subject to change as more information becomes available; the latest information, including answers to commonly asked questions, can be found online (http:// www.cdc.gov/zika). Pediatric health care providers should work closely with obstetric providers to identify infants whose mothers were potentially infected with Zika virus during pregnancy (based on travel to or residence in an area with Zika virus transmission [http://wwwnc.cdc.gov/travel/notices]), and review fetal ultrasounds and maternal testing for Zika virus infection (see Interim Guidelines for Pregnant Women During a Zika Virus Outbreak*) (1). Zika virus testing is recommended for 1) infants with microcephaly or intracranial calcifications born to women who traveled to or resided in an area with Zika virus transmission while pregnant; or 2 ) infants born to mothers with positive or inconclusive test results for Zika virus infection. For infants with laboratory evidence of a possible congenital Zika virus infection, additional clinical evaluation and follow-up is recommended. Health care providers should contact their state or territorial health department to facilitate testing. As an arboviral disease, Zika virus disease is a nationally notifiable condition.

Zika virus is a mosquito-borne flavivirus primarily transmitted by Aedes aegypti mosquitoes (2,3). Aedes albopictus mosquitoes also might transmit the virus. Ae. aegypti and Ae. albopictus mosquitoes are found throughout much of the Region of the Americas, including parts of the United States, and also transmit dengue and chikungunya viruses (4). Zika virus infections have also been documented through both intrauterine transmission resulting in congenital infection and intrapartum transmission from a viremic mother to her newborn $(5,6)$. Zika virus RNA has been detected in breast milk, but Zika virus transmission through breastfeeding has not been documented (5).

\footnotetext{
*http://www.cdc.gov/mmwr/volumes/65/wr/mm6502e1.htm.
}

During outbreaks, humans are the primary host for Zika virus. An estimated $80 \%$ of persons infected with Zika virus are asymptomatic $(2,7)$. Symptomatic disease generally is mild and characterized by acute onset of fever, maculopapular rash, arthralgia, or nonpurulent conjunctivitis. Symptoms typically last from several days to 1 week. Based on information from previous outbreaks, severe disease requiring hospitalization is uncommon and fatalities are rare $(6,7)$. During the current outbreak in Brazil, Zika virus RNA has been identified in specimens (i.e., brain tissue, placenta, and amniotic fluid) from several infants with microcephaly and from fetal losses in women infected with Zika virus during pregnancy $(6,8,9)$. The Brazil Ministry of Health has reported a marked increase from previous years in the number of infants born with microcephaly and intracranial calcifications in 2015, although it is not known how many of these cases are associated with Zika virus infection $(6,8-11)$.

\section{Zika Virus Testing Considerations and Classification}

The diagnosis of Zika virus infection is made through molecular and serologic testing (2). This includes reverse transcription-polymerase chain reaction (RT-PCR) for viral RNA, and immunoglobulin (Ig) M ELISA and plaque reduction neutralization test (PRNT) for Zika virus antibodies. Because it is currently not known which type of testing most reliably establishes the diagnosis of congenital infection, CDC recommends both molecular and serologic testing of infants who are being evaluated for evidence of a congenital Zika virus infection (Box 1). No commercial tests for Zika virus are available; Zika virus testing is performed at CDC and some state and territorial health departments. Health care providers should contact their state or territorial health department to facilitate testing.

Zika virus RT-PCR testing should be performed on serum specimens collected from the umbilical cord or directly from the infant within 2 days of birth (12). In addition, cerebrospinal fluid (CSF) obtained for other studies, and frozen and fixed placenta obtained at delivery, should also be tested by RT-PCR. IgM ELISA for Zika virus and dengue virus should be performed on infant serum, infant CSF, and maternal serum; however, results of these assays can be falsely positive because of cross-reacting antibodies $(9,12)$. PRNT can be 
performed to measure virus-specific neutralizing antibodies and to discriminate between cross-reacting antibodies from closely related flaviviruses (e.g., dengue or yellow fever viruses). Finally, immunohistochemical staining to detect Zika virus antigen on fixed placenta and umbilical cord tissues can be considered.

An infant is considered congenitally infected if Zika virus RNA or viral antigen is identified in any of the samples submitted, including testing of amniotic fluid and testing of the placenta or umbilical cord. In addition, Zika virus IgM antibodies with confirmatory neutralizing antibody titers that are $\geq 4$-fold higher than dengue virus neutralizing antibody titers in the infant serum or CSF constitute evidence of a congenital Zika virus infection. If $\mathrm{Zika}$ virus neutralizing antibody titers are $<4$-fold higher than dengue, results are considered inconclusive.

\section{Recommendations for Infants with Microcephaly or Intracranial Calcifications Detected Prenatally or at Birth Whose Mothers Were Potentially Infected with Zika Virus During Pregnancy}

For the purpose of evaluating an infant for possible congenital Zika virus infection, microcephaly is defined as occipitofrontal circumference less than the third percentile, based on standard growth charts (e.g., Fenton, Olsen, CDC, or WHO growth curves) for sex, age, and gestational age at birth (13). For a diagnosis of microcephaly to be made, the occipitofrontal circumference should be disproportionately small in comparison with the length of the infant and not explained by other etiologies (e.g., other congenital disorders). If an infant's occipitofrontal circumference is equal to or greater than the third percentile but is notably disproportionate to the length of the infant, or if the infant has deficits that are related to the central nervous system, additional evaluation for Zika virus infection might be considered.

When an infant is born with microcephaly or intracranial calcifications to a mother who was potentially infected with Zika virus during pregnancy, the infant should be tested for Zika virus infection (Figure 1) (Box 1). In addition, further clinical evaluation and laboratory testing is recommended for the infant (Box 2). The mother should also be tested for a Zika virus infection, if this testing has not already been performed during pregnancy. An ophthalmologic evaluation, including retinal examination, should occur during the first month of life, given reports of abnormal eye findings in infants with possible congenital Zika virus infection (11).

For infants with any positive or inconclusive test findings for Zika virus infection, health care providers should report the case to the state, territorial, or local health department and assess the infant for possible long-term sequelae (Box 3). This includes a repeat hearing screen at age 6 months, even if
BOX 1. Recommended Zika virus laboratory testing for infants when indicated*

- Test infant serum for Zika virus RNA, Zika virus immunoglobulin (Ig) M and neutralizing antibodies, and dengue virus IgM and neutralizing antibodies. The initial sample should be collected either from the umbilical cord or directly from the infant within 2 days of birth, if possible.

- If cerebrospinal fluid is obtained for other studies, test for Zika virus RNA, Zika virus IgM and neutralizing antibodies, and dengue virus $\operatorname{IgM}$ and neutralizing antibodies.

- Consider histopathologic evaluation of the placenta and umbilical cord with Zika virus immunohistochemical staining on fixed tissue and Zika virus RT-PCR on fixed and frozen tissue.

- If not already performed during pregnancy, test mother's serum for Zika virus IgM and neutralizing antibodies, and dengue virus $\operatorname{IgM}$ and neutralizing antibodies.

* Indications for testing include 1) infants with microcephaly or intracranial calcifications born to women who traveled to or resided in an area with Zika virus transmission while pregnant, or 2) infants born to mothers with positive or inconclusive test results for Zika virus infection.

the initial hearing screening test was normal, because of the potential for delayed hearing loss as has been described with other infections such as cytomegalovirus (14).

For infants with microcephaly or intracranial calcifications who have negative results on all Zika virus tests performed, health care providers should evaluate for other possible etiologies and treat as indicated.

\section{Recommendations for Infants without Microcephaly or Intracranial Calcifications Whose Mothers Were Potentially Infected with Zika Virus During Pregnancy}

For an infant without microcephaly or intracranial calcifications born to a mother who was potentially infected with Zika virus during pregnancy, subsequent evaluation is dependent on results from maternal Zika virus testing (Figure 2). If the test results for the mother were negative for Zika virus infection, the infant should receive routine care (e.g., newborn metabolic and hearing screens). If the mother received positive or inconclusive results of tests for Zika virus infection, the infant should be tested for a possible congenital Zika virus infection (Box 1). If the results of all of the infant's tests are negative for evidence of Zika virus infection, then no further Zika virus testing and evaluation is recommended. If any of the infant's samples test positive or inconclusive, then the infant should undergo further clinical evaluation (Box 2). The infant should also be 
FIGURE 1. Interim guidelines for the evaluation and testing of infants with microcephaly* or intracranial calcifications whose mothers traveled to or resided in an area with Zika virus transmission ${ }^{\dagger}$ during pregnancy $\$$

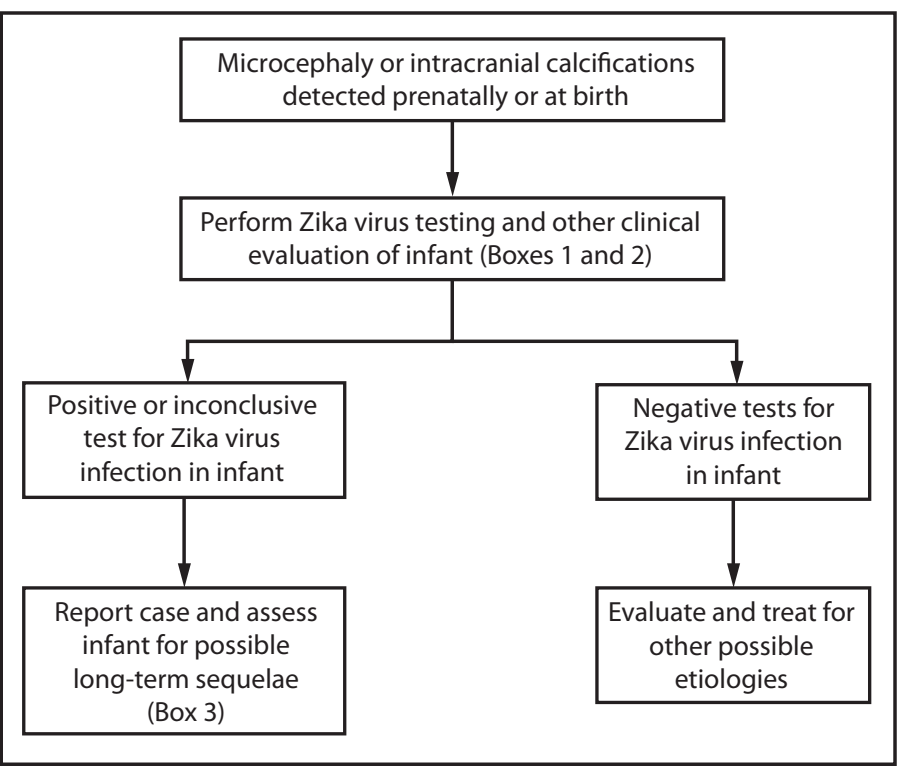

* Microcephaly defined as occipitofrontal circumference less than the third percentile for gestational age and sex not explained by other etiologies.

† Areas with Zika virus transmission are listed on CDC's webpage. http://wwwnc. cdc.gov/travel/notices.

$\S$ Laboratory evidence of Zika virus infection includes 1) detectable Zika virus, Zika virus RNA, or Zika virus antigen in any clinical sample, or 2) positive Zika virus immunoglobulin $M$ with confirmatory neutralizing antibody titers that are $\geq 4$-fold higher than dengue virus neutralizing antibody titers in serum or cerebrospinal fluid. Testing would be considered inconclusive if Zika virus neutralizing antibody titers are $<4$-fold higher than dengue virus neutralizing antibody titers.

followed to assess for possible long-term sequelae (Box 3), and the infant's case should be reported to the state, territorial, or local health department. Infant follow-up should include a cranial ultrasound to assess for subclinical findings, unless prenatal ultrasound results from the third trimester demonstrated no abnormalities of the brain. Ophthalmologic examination and a repeat hearing screen are also recommended, as previously described for infants with microcephaly or intracranial calcifications. Developmental monitoring and screening during the first year of life is recommended for all children with congenital Zika virus infection.

If the mother has not undergone any previous testing for Zika virus infection during pregnancy, $\mathrm{CDC}$ recommends that she receive testing only if she reported symptoms consistent with Zika virus disease during or within 2 weeks of any time spent in an area with ongoing Zika virus transmission while she was pregnant $(1,15)$. If the mother has any positive or inconclusive findings from tests for Zika virus infection, then the infant should undergo testing for evidence of a congenital Zika virus infection (Box 1). If the mother has not received any
BOX 2. Recommended clinical evaluation and laboratory testing for infants with possible congenital Zika virus infection

For all infants with possible congenital Zika virus infection, perform the following:

- Comprehensive physical examination, including careful measurement of the occipitofrontal circumference, length, weight, and assessment of gestational age.

- Evaluation for neurologic abnormalities, dysmorphic features, splenomegaly, hepatomegaly, and rash or other skin lesions. Full body photographs and any rash, skin lesions, or dysmorphic features should be documented. If an abnormality is noted, consultation with an appropriate specialist is recommended.

- Cranial ultrasound, unless prenatal ultrasound results from third trimester demonstrated no abnormalities of the brain.

- Evaluation of hearing by evoked otoacoustic emissions testing or auditory brainstem response testing, either before discharge from the hospital or within 1 month after birth. Infants with abnormal initial hearing screens should be referred to an audiologist for further evaluation.

- Ophthalmologic evaluation, including examination of the retina, either before discharge from the hospital or within 1 month after birth. Infants with abnormal initial eye evaluation should be referred to a pediatric ophthalmologist for further evaluation.

- Other evaluations specific to the infant's clinical presentation.

\section{For infants with microcephaly or intracranial} calcifications, additional evaluation includes the following:

- Consultation with a clinical geneticist or dysmorphologist.

- Consultation with a pediatric neurologist to determine appropriate brain imaging and additional evaluation (e.g., ultrasound, computerized tomography scan, magnetic resonance imaging, and electroencephalogram).

- Testing for other congenital infections such as syphilis, toxoplasmosis, rubella, cytomegalovirus infection, lymphocytic choriomeningitis virus infection, and herpes simplex virus infections. Consider consulting a pediatric infectious disease specialist.

- Complete blood count, platelet count, and liver function and enzyme tests, including alanine aminotransferase, aspartate aminotransferase, and bilirubin.

- Consideration of genetic and other teratogenic causes based on additional congenital anomalies that are identified through clinical examination and imaging studies. 
BOX 3. Recommended long-term follow-up for infants with possible congenital Zika virus infection

- Report case to state, territorial, or local health department and monitor for additional guidance as it is released.

- Conduct additional hearing screen at age 6 months, plus any appropriate follow-up of hearing abnormalities detected through newborn hearing screening.

- Carefully evaluate occipitofrontal circumference and developmental characteristics and milestones throughout the first year of life, with use of appropriate consultations with medical specialists (e.g., pediatric neurology, developmental and behavioral pediatrics, physical and speech therapy).

previous testing for Zika virus, and did not report clinical illness consistent with Zika virus disease during pregnancy, no further testing of the mother or infant is recommended (Figure 2).

\section{Management and Prevention of Congenital Zika Virus Infections}

No specific antiviral treatment is available for Zika virus infections and no vaccine against Zika virus is available (2). Treatment of congenital Zika virus infection is supportive and should address specific medical and neurodevelopmental issues for the infant's particular needs; investigations are ongoing to better understand what services will be most effective for these children as they grow (16). Mothers are encouraged to breastfeed infants even in areas where Zika virus is found, as available evidence indicates the benefits of breastfeeding outweigh any theoretical risks associated with Zika virus infection transmission through breast milk $(5,17)$.

The only way to prevent congenital Zika virus infection is to prevent maternal infection, either by avoiding areas where Zika virus transmission is ongoing or strictly following steps to avoid mosquito bites $(15,18)$. Mosquito-bite prevention includes using air conditioning or window and door screens when indoors, wearing long sleeves and pants, using permethrin-treated clothing and gear, and using insect repellents. When used according to the product label, U.S. Environmental Protection Agency-registered insect repellents are safe for pregnant women (18).

\footnotetext{
${ }^{1}$ Division of Vector-Borne Diseases, National Center for Emerging and Zoonotic Infectious Diseases, CDC; ${ }^{2}$ Division of Human Development and Disability, National Center on Birth Defects and Developmental Disabilities, CDC; ${ }^{3}$ Division of Birth Defects and Developmental Disabilities, National Center on Birth Defects and Developmental Disabilities, CDC; ${ }^{4}$ Division of Public Health Information Dissemination, Center for Surveillance, Epidemiology, and Laboratory Services, CDC.
}

Corresponding author: Cynthia Moore, ZikaMCH@cdc.gov, 404-639-3286.

\section{References}

1. Petersen EE, Staples JE, Meaney-Delman D, et al. Interim guidelines for pregnant women during a Zika virus outbreak-United States, 2016. MMWR Morb Mortal Wkly Rep 2016;65:30-3. http://dx.doi. org/10.15585/mmwr.mm6502e1.

2. Hayes EB. Zika virus outside Africa. Emerg Infect Dis 2009;15:1347-50. http://dx.doi.org/10.3201/eid1509.090442.

3. CDC. Zika virus. Atlanta, GA: US Department of Health and Human Services, CDC; 2016. http://www.cdc.gov/zika/index.html.

4. CDC. Chikungunya virus: surveillance and control of Aedes aegypti and Aedes albopictus in the United States. Atlanta, GA: US Department of Health and Human Services, CDC; 2015. http://www.cdc.gov/ chikungunya/resources/vector-control.html.

5. Besnard M, Lastere S, Teissier A, Cao-Lormeau V, Musso D. Evidence of perinatal transmission of Zika virus, French Polynesia, December 2013 and February 2014. Euro Surveill 2014;19:20751. http://dx.doi. org/10.2807/1560-7917.ES2014.19.13.20751.

6. European Centre for Disease Prevention and Control. Rapid risk assessment: Zika virus epidemic in the Americas: potential association with microcephaly and Guillain-Barré syndrome. Stockholm, Sweden: European Centre for Disease Prevention and Control; 2015. http://ecdc. europa.eu/en/publications/Publications/zika-virus-americas-associationwith-microcephaly-rapid-risk-assessment.pdf.

7. Duffy MR, Chen TH, Hancock WT, et al. Zika virus outbreak on Yap Island, Federated States of Micronesia. N Engl J Med 2009;360:2536-43. http://dx.doi.org/10.1056/NEJMoa0805715.

8. Oliveira Melo AS, Malinger G, Ximenes R, Szejnfeld PO, Alves Sampaio $S$, Bispo de Filippis AM. Zika virus intrauterine infection causes fetal brain abnormality and microcephaly: tip of the iceberg? Ultrasound Obstet Gynecol 2016;47:6-7. http://dx.doi.org/10.1002/uog.15831.

9. Hennessey M, Fischer M, Staples JE. Zika virus spreads to new areasRegion of the Americas, May 2015-January 2016. MMWR Morb Mortal Wkly Rep 2016;65:1-4. http://dx.doi.org/10.15585/mmwr. mm6503eler.

10. Schuler-Faccini L, Ribeiro EM, Feitosa IM, et al. Possible association between Zika virus infection and microcephaly-Brazil, 2015. MMWR Morb Mortal Wkly Rep 2016;65:1-4. http://dx.doi.org/10.15585/ mmwr.mm6503e2er.

11. Ventura CV, Maia M, Bravo-Filho V, Góis AL, Belfort R Jr. Zika virus in Brazil and macular atrophy in a child with microcephaly. Lancet 2016;387:228. http://dx.doi.org/10.1016/S0140-6736(16)00006-4.

12. Lanciotti RS, Kosoy OL, Laven JJ, et al. Genetic and serologic properties of Zika virus associated with an epidemic, Yap State, Micronesia, 2007. Emerg Infect Dis 2008;14:1232-9. http://dx.doi.org/10.3201/eid1408.080287.

13. World Health Organization. WHO child growth standards: length/ height-for-age, weight-for-age, weight-for-height and body mass indexfor-age: methods and development. Geneva, Switzerland: World Health Organization; 2006. http://www.who.int/childgrowth/publications/ technical_report_pub/en/index.html.

14. Dahle AJ, Fowler KB, Wright JD, Boppana SB, Britt WJ, Pass RF. Longitudinal investigation of hearing disorders in children with congenital cytomegalovirus. J Am Acad Audiol 2000;11:283-90.

15. CDC. Travel notices. Atlanta, GA: US Department of Health and Human Services, CDC; 2016. http://wwwnc.cdc.gov/travel/notices.

16. CDC. Developmental disabilities. Atlanta, GA: US Department of Health and Human Services; 2015. http://www.cdc.gov/ncbddd/ developmentaldisabilities/index.html.

17. American Academy of Pediatrics Section on Breastfeeding. Breastfeeding and the use of human milk.[Policy statement]. Pediatrics 2005;115:496-506. http://dx.doi.org/10.1542/peds.2004-2491.

18. Nasci RS, Wirtz RA, Brogdon WG. Protection against mosquitoes, ticks, and other arthropods. In: CDC health information for international travel, 2016. New York, NY: Oxford University Press; 2015:94-9. http:// wwwnc.cdc.gov/travel/yellowbook/2016/the-pre-travel-consultation/ protection-against-mosquitoes-ticks-other-arthropods. 
FIGURE 2. Interim guidelines for the evaluation and testing of infants without microcephaly* or intracranial calcifications whose mothers traveled to or resided in an area with Zika virus transmission ${ }^{\dagger}$ during pregnancy ${ }^{\S, n, * *}$

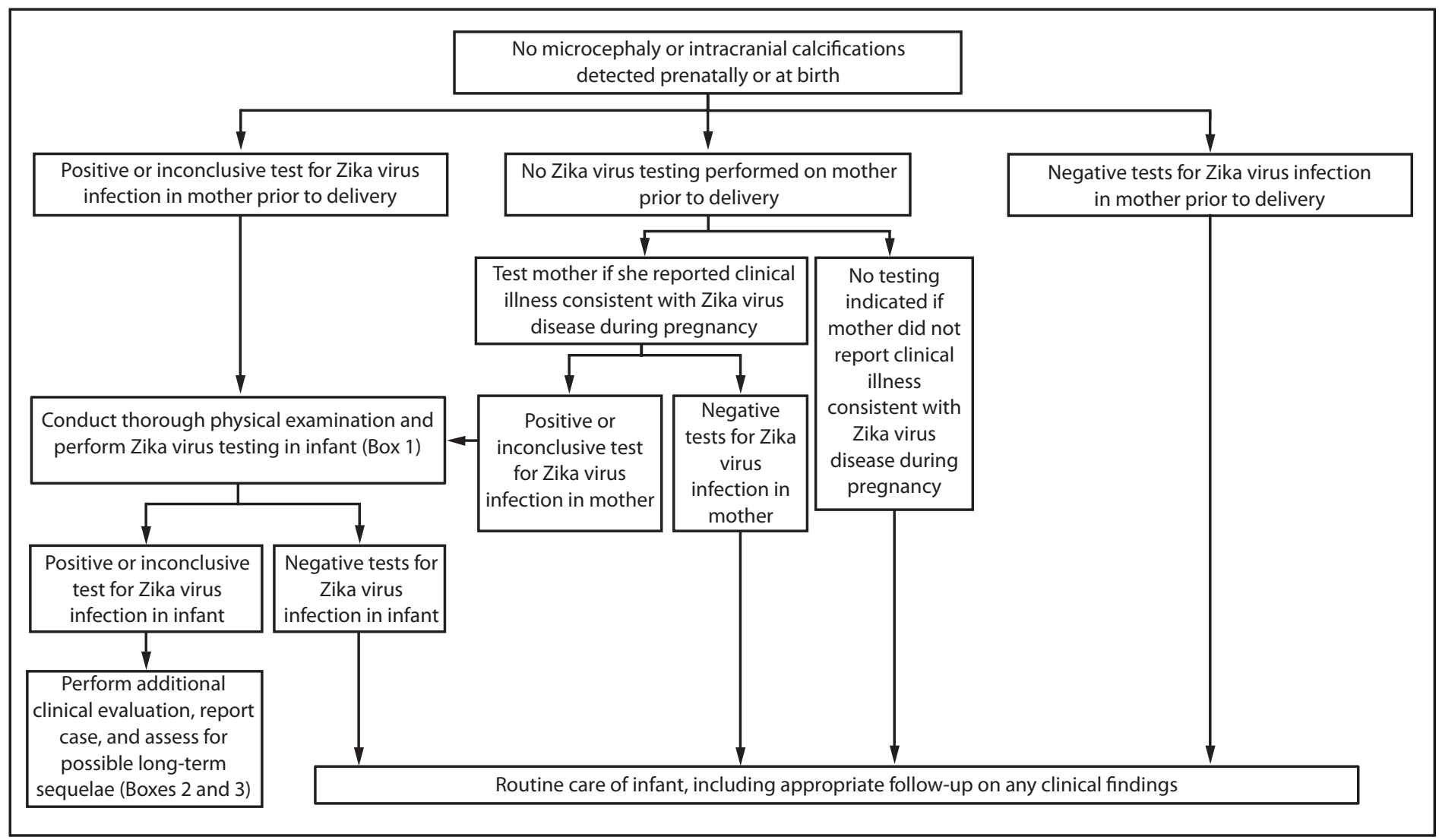

* Microcephaly defined as occipitofrontal circumference less than the third percentile for gestational age and sex not explained by other etiologies.

† Areas with Zika virus transmission are listed on CDC's webpage. http://wwwnc.cdc.gov/travel/notices.

$\S$ Laboratory evidence of Zika virus infection includes 1) detectable Zika virus, Zika virus RNA, or Zika virus antigen in any clinical sample, or 2) positive Zika virus Immunoglobulin M (IgM) with confirmatory neutralizing antibody titers that are $\geq 4$-fold higher than dengue virus neutralizing antibody titers in serum or cerebrospinal fluid. Testing would be considered inconclusive if Zika virus neutralizing antibody titers are $<4$-fold higher than dengue virus neutralizing antibody titers.

9 If mother reported clinical illness consistent with Zika virus disease during pregnancy and testing is indicated, perform Zika virus reverse transcription-polymerase chain reaction testing on serum specimen collected $\leq 7$ days after illness onset when possible. Perform Zika and dengue virus IgM and neutralizing antibodies on serum specimens collected $\geq 4$ days after illness onset.

** Clinical illness is consistent with Zika virus disease if two or more symptoms (including acute onset of fever, maculopapular rash, arthralgia, or conjunctivitis) are present during or within 2 weeks of any time spent in an area with ongoing Zika virus transmission. 